\title{
A Simple Fuzzy PI Control of Dual-Motor Driving Servo System
}

\author{
Haibo Zhao ${ }^{1,2, a}$, Chengguang Wang ${ }^{3}$ \\ ${ }^{1}$ Engineering Technology Research Center of Optoelectronic Appliance, Tongling University, Tongling Anhui, \\ 244061, China \\ ${ }^{2}$ Department of Electrical Engineering, Tongling University, Tongling Anhui, 244061, China \\ ${ }^{3}$ Sichuan Institute of Aerospace System Engineering, Chengdu Sichuan, 610100, China
}

\begin{abstract}
Aiming at the control problem of dual-motor driving servo system, in order to avoid the complicated numerical calculation of complicated control algorithm, we presented a simple fuzzy PI controller for the first time. Firstly, we explained system control principle. In view of the shortcomings of current-error negative feedback, we used speed-error negative feedback synchronous driving control strategy. Then we theoretically analyzed dual-motor synchronous driving system in detail. We proposed the conditions of dual-motor driving servo system synchronous operation for step signal and ramp signal, respectively. Finally, we presented a simple fuzzy PI controller, by combining fuzzy controller with PI controller. We analyzed the fuzzy PI control system and found it was stable. We considered two cases in simulation tests comparing fuzzy PI control with PI control. Simulation results show that fuzzy PI control has better adaptability than conventional PI control, validating the effectiveness and efficiency of the proposed control strategy.
\end{abstract}

\section{Introduction}

Dual-motor driving servo system is widely used in military, civilian and other occasions. Many scholars have studied it. In [1,2], the mechanical model of the rotary kiln drive as well as the development of an appropriate laboratory simulating system were presented. Experimental results proved that gear faults can be identified by monitoring the motor phase current as well as the frequency spectrum of this current. In [3], the dynamic model of dual-motor driving system was given, using switching toeque control, simulation results showed that system has good position control precision. In [4], simulation model based on the adaptive full-order flux observer and torque sensor vector control was established to observe or test variety of system dynamic response directly. However, all the above mentioned results have used more complicated intelligent control strategy.

Fuzzy control algorithm has a high maturity and has been widely used in many occasions. Quang Hieu Ngo, et al. [5] presented a fuzzy sliding mode control strategy for container cranes. Simulation results illustrated the efficiency of the proposed control law. Chun-Yan Wang, et al. [6] provided a common adaptive fuzzy smooth dynamic controller for a class of uncertain switched nonlinear systems. Its effectiveness and feasibility were demonstrated by both a numerical example and a chemical system. Do Wan Kim, et al. [7] presented a new direct discrete-time design methodology of

\footnotetext{
${ }^{a}$ Corresponding author : happyzhaohaibo@126.com
} 
a robust sampled-data fuzzy controller for a class of nonlinear systems. The simulation results for the sampled-data depth control of AUV convincingly illustrated the effectiveness of the developed techniques. Xiaojie Su, et al. [8] presented a T-S model-based fuzzy controller design approach for electromagnetic suspension systems. The T-S fuzzy model was firstly applied to represent the nonlinear electromagnetic suspension systems. Numerical simulation on an electromagnetic suspension system was performed to validate the effectiveness of the proposed approach. From [5-8], we can see that fuzzy control is rarely used alone, and is usually used in conjunction with other control methods.

Fuzzy control is introduced into the logical reasoning, has strong adaptive ability and a good control effect on complex system, but separate fuzzy control cannot fundamentally eliminate steadystate error, control precision is low [9]. Conventional PI control principle is simple, easy to implement, has been widely used in industrial process control. But the coefficient setting of proportion and integral is a very troublesome thing, reasonable PI parameters are usually determined online by experienced technical personnel. In the case of the control object's time variant, a set of fixed PI parameters cannot meet the requirements of system. Fuzzy PI control algorithm is formed by combining fuzzy control with conventional PI control.

In recent years, a simple fuzzy PI control has rarely been used in dual-motor driving servo system. Hence, it is imperative to study the fuzzy PI control of dual-motor driving servo system. This algorithm can not only achieve precise control, but also has strong adaptability. It can automatically modify the control parameters of PI according to the changes of the environment.

This paper is organized as follows: system modeling is given in Section 2. System control principle is presented in Section 3. The theoretic analysis of dual-motor synchronous driving system is given in Section 4. Section 5 presents the design of fuzzy PI controller. Simulation analysis in Section 6 to show the effectiveness of fuzzy PI control strategy. Section 7 concludes this paper.

\section{System Control Principle}

Since the system is a dual-motor driving mode, it must be ensured that the speeds of the two motors are synchronous. In the ideal case, if the two motors are the same, for the same command signal, the output speeds must be the same. However, due to the discrete characteristic of motors and other components, even if the same tag parameters of components, the actual parameters cannot be completely consistent. Therefore, it is necessary to solve the problem of dual-motor synchronous driving, otherwise there will be a so-called "servo fight" phenomenon.

The existing method is to use "current-error negative feedback", which can achieve the synchronous driving of two motors by the balanced control of current. Because the motor parameters cannot be completely consistent, even if the consistent motor current may not be able to achieve the same speed, and the effect of synchronous driving must be based on the speed synchronization. In view of the shortcomings of current-error negative feedback, we use "speed-error negative feedback" synchronous driving control strategy.

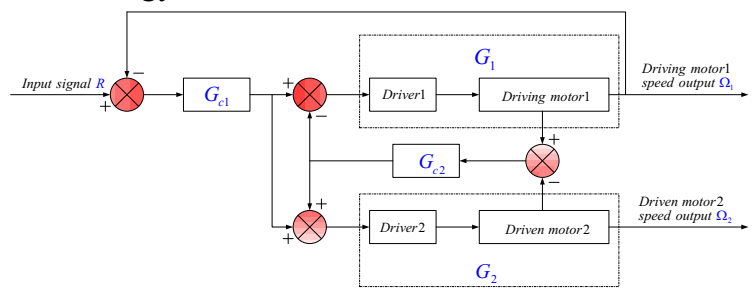

Figure 1. Control block diagram of dual-motor synchronous driving.

As is shown in Fig. 1, take one of the motors as driving motor, retaining its speed loop, the other is driven motor, disconnecting the speed loop. For each motor, the input current instruction is a composite signal of the basic current given signal and speed-error feedback signal. And the speederror negative feedback signals of two motors are equal, but the polarities are opposite. 
Where $G_{C 1}$ is speed loop controller(type-PI), $G_{1}$ and $G_{2}$ are transfer functions between servo motor 1, 2 and respective current loop. $G_{C 2}$ is fuzzy PI controller, $R$ is speed input signal. $\Omega_{1}$ and $\Omega_{2}$ are speed output of motor 1 and 2 , respectively.

In a dynamic process, if the two motor's speeds are not synchronous, after speed-error was linear amplified, feedback to the two motors current given end respectively as auxiliary input. Because the current loop response is much faster than the speed loop response, the speed-error signal is introduced into the current given end to inhibit the two motors speed out of synchronization as soon as possible. So when the speed of motor 1 is faster than the speed of motor 2, the given input of motor 1 will slightly decrease on the basis of basic current, and the given input of motor 2 will increase and vice versa.

\section{Theoretic Analysis of Dual-Motor Synchronous Driving System}

Dual-motor synchronous driving control block diagram, as shown in fig. 1. Using Mason formula, we can solve the transfer function between $\Omega_{1}$ and $R$

$$
G_{Y 1-R_{-} C L}=\frac{\mathrm{W}_{1}(s)}{R(s)}=\frac{G_{1} G_{C 1}+2 G_{1} G_{2} G_{C 1} G_{C 2}}{\mathrm{D}}
$$

where $\mathrm{D}=1+G_{C 1} G_{1}+G_{C 2} G_{1}+G_{C 2} G_{2}+2 G_{C 1} G_{C 2} G_{1} G_{2}$

Similarly, the transfer function between $\Omega_{2}$ and $R$

$$
G_{Y 2-R_{-} C L}=\frac{\mathrm{W}_{2}(s)}{R(s)}=\frac{G_{C 1} G_{2}+2 G_{1} G_{2} G_{C 1} G_{C 2}}{\mathrm{D}}
$$

Then the transfer function between $\Omega_{1}-\Omega_{2}$ and $R$

$$
\frac{\mathrm{W}_{1}(s)-\mathrm{W}_{2}(s)}{R(s)}=\frac{G_{C 1}\left(G_{1}-G_{2}\right)}{\mathrm{D}}
$$

From Eq. (11), we can see that when the two motors are exactly the same, namely $G_{1}=G_{2}$, regardless of the input signal, regardless of whether there is a speed-error negative feedback or not, $\Omega_{1}-\Omega_{2}=0$, the two motors' speed must be synchronized(assume there is no disturbance).

Eqs. (1), (2), (3) are close loop transfer functions, the equivalent open-loop transfer functions of the unit feedback are

$$
\begin{gathered}
G_{Y 1-R_{-} O P}=\frac{G_{Y 1-R_{-} C L}}{1-G_{Y 1-R_{-} C L}}=\frac{G_{C 1} G_{1}\left(1+2 G_{C 2} G_{2}\right)}{1+G_{C 2} G_{1}+G_{C 2} G_{2}} \\
G_{Y 2-R_{-} O P}=\frac{G_{Y 2-R_{-} C L}}{1-G_{Y 2-R_{-} C L}}=\frac{G_{C 1} G_{2}\left(1+2 G_{C 2} G_{1}\right)}{1+G_{C 1} G_{1}+G_{C 2} G_{1}+G_{C 2} G_{2}-G_{C 1} G_{2}} \\
G_{\mathrm{D} Y-R-O P}=\frac{G_{\mathrm{D} Y-R_{-} C L}}{1-G_{\mathrm{D} Y-R_{-} C L}}=\frac{G_{c 1}\left(G_{1}-G_{2}\right)}{1+G_{C 1} G_{2}+G_{C 2} G_{1}+G_{C 2} G_{2}+2 G_{C 1} G_{C 2} G_{1} G_{2}}
\end{gathered}
$$

From fig.1, we can get the transfer function between current loop and servo motor as follows:

$$
G_{I}^{\prime}(s)=\frac{K_{M} K_{I} T_{I} s+K_{M}}{C_{1}^{\prime} s^{3}+C_{2}^{\prime} s^{2}+C_{3}^{\prime} s+\alpha D_{M}+\alpha D_{L}}
$$

Where,

$$
\begin{gathered}
C_{1}^{\prime}=L T_{I} J_{m}+L T_{I} J_{L}, C_{2}^{\prime}=R T_{I} J_{m}+R T_{I} J_{L}+L T_{I} D_{M}+L T_{I} D_{L}+\alpha K_{I} T_{I} J_{m}+\alpha K_{I} T_{I} J_{L}, \\
C_{3}^{\prime}=R T_{I} D_{M}+R T_{I} D_{L}+\alpha K_{I} T_{I} D_{M}+\alpha K_{I} T_{I} D_{L}+\alpha J_{m}+\alpha J_{L}+K_{M} K_{\Omega} T_{I}
\end{gathered}
$$

For convenience of analysis, let 


$$
\begin{aligned}
& G_{1}=\frac{m_{1} s+n_{1}}{a_{1} s^{3}+b_{1} s^{2}+c_{1} s+d_{1}} \quad, \quad G_{2}=\frac{m_{2} s+n_{2}}{a_{2} s^{3}+b_{2} s^{2}+c_{2} s+d_{2}} \\
& G_{C 1}=\frac{K_{P 1} s+K_{I 1}}{s} \quad, \quad G_{C 2}=\frac{K_{P 2} s+K_{I 2}}{s}
\end{aligned}
$$

Where, $m_{1}, m_{2}, n_{1}, n_{2}, a_{1}, a_{2}, b_{1}, b_{2}, c_{1}, c_{2}, d_{1}$ and $d_{2}$ are all greater than zero, $K_{I 1}>0$, when using proportional feedback $K_{I 2}=0$, when using proportional integral feedback $K_{I 2}>0$, substitute Eq.(8) into Eqs.(4), (5) and (6) respectively, we get:

$$
\left\{\begin{array}{l}
G_{Y 1-R_{-} O P}=\frac{\left(m_{1} s+n_{1}\right)\left(K_{P 1} s+K_{I 1}\right)\left(C_{1} s^{4}+C_{2} s^{3}+C_{3} s^{2}+C_{4} s+2 n_{2} K_{I 2}\right)}{s\left(C_{5} s^{7}+C_{6} s^{6}+C_{7} s^{5}+C_{8} s^{4}+C_{9} s^{3}+C_{10} s^{2}+C_{11} s+K_{I 2} n_{1} d_{2}+K_{I 2} n_{2} d_{1}\right)} \\
G_{Y 2-R_{-} O P}=\frac{\left(m_{2} s+n_{2}\right)\left(K_{P 1} s+K_{I 1}\right)\left(C_{12} s^{4}+C_{13} s^{3}+C_{14} s^{2}+C_{15} s+2 n_{1} K_{I 2}\right)}{s\left(C_{16} s^{7}+C_{17} s^{6}+C_{18} s^{5}+C_{19} s^{4}+C_{20} s^{3}+C_{21} s^{2}+C_{22} s+K_{I 2} n_{1} d_{2}+K_{I 2} n_{2} d_{1}+K_{I 1} n_{1} d_{2}-K_{I 1} n_{2} d_{1}\right)} \\
G_{\Delta Y-R-O P}=\frac{s\left(K_{P 1} s+K_{I 1}\right)\left(C_{23} s^{4}+C_{24} s^{3}+C_{25} s^{2}+C_{26} s+n_{1} d_{2}-n_{2} d_{1}\right)}{C_{27} s^{8}+C_{28} s^{7}+C_{29} s^{6}+C_{30} s^{5}+C_{31} s^{4}+C_{32} s^{3}+C_{33} s^{2}+C_{34} s+2 K_{I 1} K_{I 2} n_{2} n_{1}}
\end{array}\right.
$$

where, $C_{i}(i=1,2,3, \ldots, 34)$ are polynomial coefficients.

There are two cases in Eq. (9):

(1). $K_{I 2}{ }^{1} 0, K_{I 2} n_{1} d_{2}+K_{I 2} n_{2} d_{1}+K_{I 1} n_{1} d_{2}-K_{I 1} n_{2} d_{1} \neq 0$

In this case, numerator constant term coefficient of $G_{Y 1-R_{-} O P}$ is not equal to zero, constant term coefficient in denominator bracket is also not equal to zero, so $G_{Y 1-R_{-} O P}$ is type-1 system, $G_{Y 2-R_{-} O P}$ is also type-1 system, the system can be synchronized for step signal; if it also meets $n_{1} d_{2}=n_{2} d_{1}$, the above two systems output all exist steady-state error, but the steady-state error is equal, so the two systems can still be synchronized.

(2). $K_{I 2}{ }^{1} 0, K_{I 2} n_{1} d_{2}+K_{I 2} n_{2} d_{1}+K_{I 1} n_{1} d_{2}-K_{I 1} n_{2} d_{1}=0$

In this case, $G_{Y 1-R_{-} O P}$ is still type- 1 system, $G_{Y 2-R_{-} O P}$ is at least type-2 system. The two systems can be synchronized for step signal, but cannot be synchronized for ramp signal.

From the above analysis, we can see that dual-motor can be synchronized for step signal when $K_{I 2}{ }^{1} 0$, dual-motor could be synchronized for ramp signal only when it meets:

$K_{I 2}{ }^{1} 0, K_{I 2} n_{1} d_{2}+K_{I 2} n_{2} d_{1}+K_{I 1} n_{1} d_{2}-K_{I 1} n_{2} d_{1} \neq 0$ and $n_{1} d_{2}=n_{2} d_{1}$.

\section{Design of Fuzzy PI Controller}

System control block diagram is shown in Fig. 2.

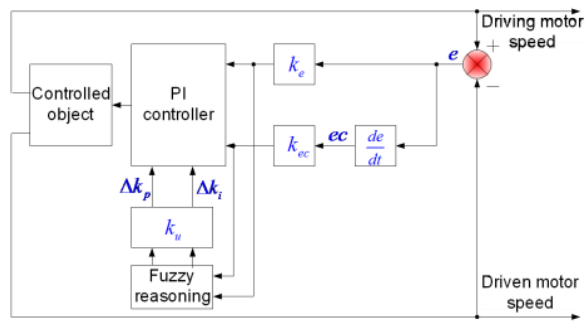

Figure 2. System control block diagram.

System control block diagram is shown in figure 2. The fuzzy PI controller use error $e$ and error change $e c$ as input. The control scheme is to find out the fuzzy relation between the two parameters of PI and $e, e c$ firstly, the two parameters of PI can be modified online by continuous testing of $e$ and $e c$, so as to meet the different control parameter requirements for different $e$ and $e c$, making the 
controlled object has a good dynamic and static performance.

\subsection{Determination of Input and Output Variables}

The speed error $e$ of dual-motor and the change rate $e c$ of speed error are as the input of fuzzy controller, correction parameters $\Delta k_{p}$ and $\Delta k_{i}$ are as the output of fuzzy controller, then PI regulator output parameters $k_{p}$ and $k_{i}$ are shown in Eq.(10).

$$
k_{p}=k_{p}^{\prime}+\Delta k_{p}, k_{i}=k_{i}^{\prime}+\Delta k_{i}
$$

where $k_{p}^{\prime}$ and $k_{i}^{\prime}$ are setting values.

\subsection{Determination of Membership Function}

Fuzzy subsets of fuzzy controller input and output variables are $e, e c, \Delta k_{p}$ and $\Delta k_{i}$ respectively. Linguistic value of each variable is [large negative, medium negative, small negative, zero, small positive, medium positive, large positive] denoted by [NB, NM, NS, ZO, PS, PM, PB], discourse domain is integer in $[-6,6]$. Taking into account the sensitivity, stability, robustness and the coverage degree of discourse domain, the membership function curve of each fuzzy subset is selected by triangle shape, shown in Fig. 3.

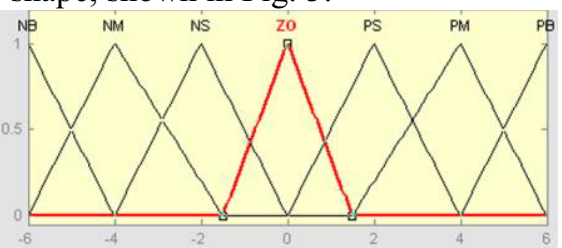

(a) Membership function of $e$ and $e c$

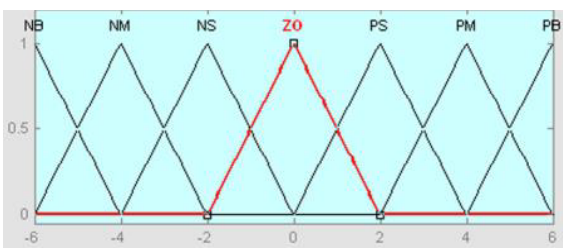

(b) Membership function of $\Delta k_{p}$ and $\Delta k_{i}$

Figure 3. Membership function curve.

\subsection{Fuzzy Control Rules}

The core of fuzzy control design is the determination of fuzzy control rules, and the selection of control rules is directly related to the performance of system. From the above parameters setting principle, through the fuzzy reasoning and experimental correction, we get the fuzzy control rules of $\Delta k_{p}$ and $\Delta k_{i}$ are shown in Table 1.

Table 1. Fuzzy control rules

\begin{tabular}{|c|c|c|c|c|c|c|c|}
\hline$e^{e c}$ & NB & $\mathrm{NM}$ & NS & $\mathrm{ZO}$ & PS & $\mathrm{PM}$ & PB \\
\hline \multirow{2}{*}{ NB } & $\mathrm{PB}$ ? & $\mathrm{PB} /$ & PM/ & $\mathrm{PM} /$ & PS/ & $\mathrm{ZO} /$ & $\mathrm{ZO} /$ \\
\hline & NB & NB & NM & NM & NS & $\mathrm{ZO}$ & $\mathrm{ZO}$ \\
\hline \multirow{2}{*}{$\mathrm{NM}$} & $\mathrm{PB} /$ & $\mathrm{PB} /$ & $\overline{\mathrm{PM} /}$ & PS/ & $\mathrm{PS} /$ & $\mathrm{ZO} /$ & $\mathrm{NS} /$ \\
\hline & NB & NB & NM & NS & NS & $\mathrm{ZO}$ & $\mathrm{ZO}$ \\
\hline \multirow{2}{*}{ NS } & PM/ & $\mathrm{PM} /$ & $\mathrm{PM}$ & PS/ & $\mathrm{ZO} /$ & $\mathrm{NS} /$ & $\mathrm{NS} /$ \\
\hline & NB & NM & NS & NS & $\mathrm{ZO}$ & PS & PS \\
\hline \multirow{2}{*}{$\mathrm{ZO}$} & $\overline{\mathrm{PM} /}$ & $\overline{\mathrm{PM} /}$ & $\mathrm{PS} /$ & $\mathrm{ZO}$ & $\mathrm{NS} /$ & $\mathrm{NM} /$ & NM/ \\
\hline & NM & NM & NS & $\mathrm{ZO}$ & PS & PM & PM \\
\hline \multirow{2}{*}{ PS } & $\overline{\mathrm{PS} /}$ & $\overline{\mathrm{PS} /}$ & $\overline{\mathrm{ZO} /}$ & $\mathrm{NS} /$ & NS & $\mathrm{NM} /$ & NM/ \\
\hline & $\mathrm{NM}$ & NS & $\mathrm{ZO}$ & PS & PS & PM & PB \\
\hline \multirow{2}{*}{ PM } & PS/ & $\mathrm{ZO} /$ & $\mathrm{NS} /$ & NM/ & $\mathrm{NM} /$ & - & $\mathrm{NB} /$ \\
\hline & $\mathrm{ZO}$ & $\mathrm{ZO}$ & PS & PS & $\mathrm{PM}$ & PB & PB \\
\hline \multirow{2}{*}{ PB } & $\mathrm{ZO} /$ & $\mathrm{ZO} /$ & $\mathrm{NM} /$ & $\mathrm{NM} /$ & NM/ & $\mathrm{NB} /$ & $\mathrm{NB} /$ \\
\hline & $\mathrm{ZO}$ & $\mathrm{ZO}$ & PS & PM & $\mathrm{PM}$ & $\mathrm{PB}$ & PB \\
\hline
\end{tabular}

\subsection{Stability of Fuzzy PI Control System}


In section 3, we have analyzed the stability conditions of PI control system in detail. In a conventional PI control system, if the linear PI controller is replaced by the nonlinear fuzzy PI controller, then the stability of the resulting control system remains unchanged [10]. Thus the stability conditions of fuzzy PI control system are the same as the PI control system. In the following simulation analysis, fuzzy PI control system is stable.

\section{Simulation Experiment}

Simulation block diagram is shown in Fig. 4:

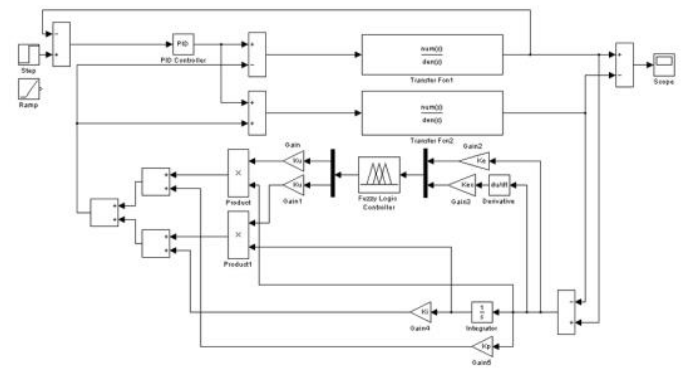

Figure 4. Simulation block diagram of fuzzy PI control.

Where, PID Controller is speed regulator, Transfer Fun 1 is transfer function between driving motor and its current loop, Transfer Fun2 is transfer function between driven motor and its current loop, the input and output of simulation block diagram are speed given signal and dual-motor speed error respectively.

According to reference[11], we get:

$$
G_{I}^{\prime}(s)=\frac{8.7318 s+6750}{1.1 \times 10^{-4} s^{3}+6.917 \times 10^{-2} s^{2}+50 s+0.85}
$$

let $G_{1}(s)=G_{I}^{\prime}(s)$

In the following simulation, system parameters are chosen as follows:

$$
k_{e}=600, k_{e c}=666.7, k_{u}=1.5, k_{p}=2.3, k_{i}=1.2
$$

PID controller parameter is chosen as follows: $\mathrm{kp}=5.6, \mathrm{ki}=1.6$

1) In order to satisfy the equation:

$$
K_{I 2} n_{1} d_{2}+K_{I 2} n_{2} d_{1}+K_{I 1} n_{1} d_{2}-K_{I 1} n_{2} d_{1}=0
$$

let

$$
G_{2}(s)=\frac{8.7318 s+6700}{1.1 \times 10^{-4} s^{3}+6.917 \times 10^{-2} s^{2}+50 s+0.8296}
$$

Thus, we get $n_{1}=6750, n_{2}=6700, d_{1}=0.85, d_{2}=0.8296$

and substituted into eq.(12), after simplification, we obtain $11295 K_{I 2}=95 K_{I 1}$.

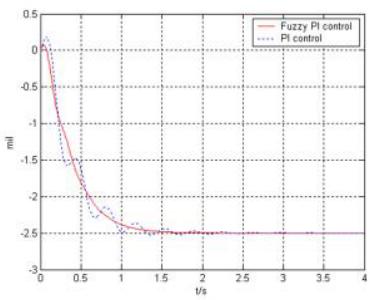

Figure 5. Speed error of dual-motor tracking ramp signal $1000 \mathrm{t}(\mathrm{mil} / \mathrm{s})$.

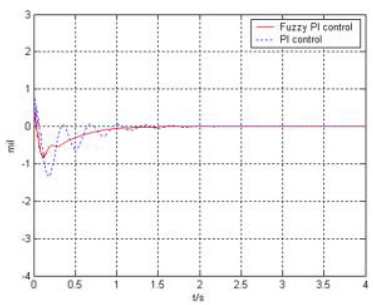

Figure 6. Speed error of dual-motor tracking step signal 500mil. 


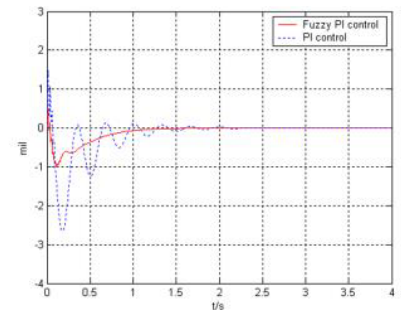

Figure 7. Speed error of dual-motor tracking step signal 1000mil.

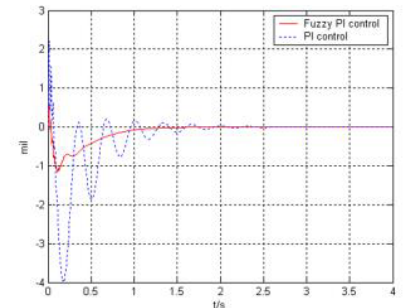

Figure 8. Speed error of dual-motor tracking step signal $1500 \mathrm{mil}$.

2) In order to satisfy the equation:

$$
K_{I 2} n_{1} d_{2}+K_{I 2} n_{2} d_{1}+K_{I 1} n_{1} d_{2}-K_{I 1} n_{2} d_{1} \neq 0
$$

and $n_{1} d_{2}=n_{2} d_{1}$, let

$$
G_{2}(s)=\frac{8.7318 s+6700}{1.1 \times 10^{-4} s^{3}+6.917 \times 10^{-2} s^{2}+50 s+0.8437}
$$

we can get $n_{1}=6750, n_{2}=6700, d_{1}=0.85, d_{2}=0.8437$ and substituted into Eq.(14), after simplification, we get $K_{I 2} \neq 0$.

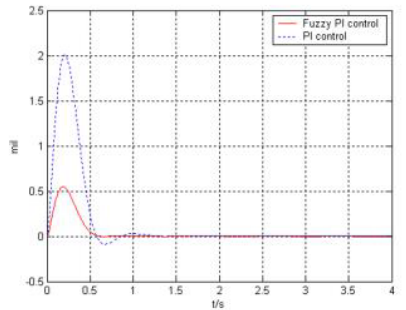

Figure 9. Speed error of dual-motor tracking ramp signal $1000 \mathrm{t}(\mathrm{mil} / \mathrm{s})$.

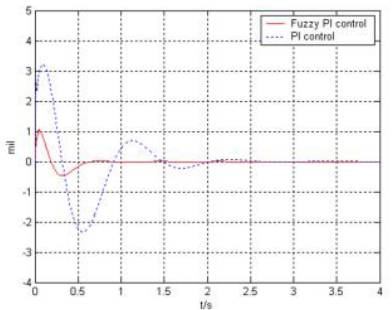

Figure 11. Speed error of dual-motor tracking step signal 1000mil.

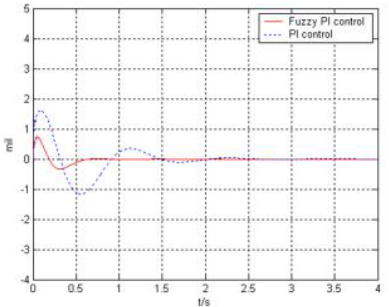

Figure 10. Speed error of dual-motor tracking step signal 500mil.

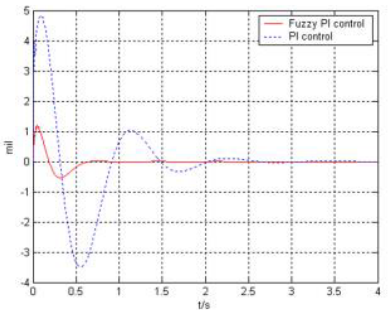

Figure 12. Speed error of dual-motor tracking step signal $1500 \mathrm{mil}$.

From Fig. 5 to Fig. 12, we can see that when the input is a ramp signal, speed-error feedback controller using fuzzy PI compared with PI, the former start up process is better, reducing the overshoot and oscillation frequency. In the condition of input step signal amplitude changes, the speed error of dual-motor changes slightly when using fuzzy PI control, but the speed error of dual-motor changes greatly when using PI control, so the fuzzy PI controller has stronger robustness than PI controller.

\section{Conclusion}

Aiming at the dual-motor driving servo system, a simple fuzzy PI controller was presented for the first time. The conventional PI control system has a large overshoot, and the pure fuzzy control cannot eliminate the steady-state error due to its structure. In this paper, the two control methods are 
combined, namely fuzzy PI control, simulation results show that the fuzzy PI control not only has better dynamic and static quality, but also has better adaptability [12-15] than conventional PI control, which can significantly improve the control performance of system, validating the efficacy of the proposed control strategy. This paper provides a reference for the further research of dual-motor driving servo system.

\section{Acknowledgment}

This work was supported by National Natural Science Foundation of China(No.61074023), Nature Science Foundation in Anhui Province of China(No.1508085MF130), Natural Science Research Key Project of Universities in Anhui Province of China (No.KJ2015A297), the Engineering Technology Research Center of Optoelectronic Appliance in Anhui Province of China, Sichuan Institute of Aerospace System Engineering in Sichuan Province of China.

The authors confirm that this article content has no conflicts of interest.

\section{References}

1. I. X. Bogiatzidis, E. D. Mitronikas, A. N. Safacas, Investigation of backlash phenomena appearing in a twin AC cement kiln drive, 19th International Conference on Electrical Machines, 2010, 1-6.

2. I. X. Bogiatzidis, A. N. Safacas, Vibration analysis and backlash identification of a twin AC drive for a cement kiln, 5th IET International Conference on Power Electronics, Machines and Drives, 2010, 1-6.

3. L. F. Sun, Research on motion characteristic and anti-backlash control of dual-motor system, The Outstanding Master's Degree Thesis of Northeastern University, June 2012.

4. H. Z. Zhu, W. X. Song, Y. Dong, Modeling and simulation of coaxial connected dual-motor drive system, Electric Mach. Control Appl. 40(2) (2013) 23-27.

5. Q. H. Ngo, N. P. Nguyen, C. N. Nguyen, Fuzzy sliding mode control of container cranes, Int. J. Control, Autom. Syst. 13(2) (2015) 419-425.

6. C. Y. Wang, X. H. Jiao, Observer-based adaptive arbitrary switching fuzzy tracking control for a class of switched nonlinear systems, Int. J. Control, Autom. Syst. 13(4) (2015) 823-830.

7. D. W. Kim, H. J. Lee, M. H. Kim, Robust sampled-data fuzzy control of nonlinear systems with parametric uncertainties: Its application to depth control of autonomous underwater vehicles, Int. J. Control, Autom. Syst. 10(6) (2015) 1164-1172.

8. X. J. Su, X. Z. Yang, P. Shi, Fuzzy control of nonlinear electromagnetic suspension systems, Mechatron. 24(4) (2014) 328-335.

9. H. Ying, S. William, J. B. James, Fuzzy control theory: a nonlinear case, Automatica, 26(3) (1990) 513-520.

10. G. R. Chen, H. Ying, Stability analysis of nonlinear fuzzy PI control systems, The Third International Conference on Industrial Fuzzy Control and Intelligent Systems, 1993, 128-133.

11. H. B. Zhao, X. H. Zhou, Research of fuzzy PI control of dual-motors driving servo system, Journal of Chaohu College, 11(3) (2009) 71-75.

12. Y. M. Jia, H. Kokame J. Lunze, Simultaneous adaptive decoupling and model matching control of a fluidized bed combustor for sewage sludge, IEEE T. Control Syst. Technol. 11(4) (2003) 571-577.

13. S. J. Yoo, Distributed adaptive consensus tracking of a class of networked non-linear systems with parametric uncertainties, IET Control Theor. Appl. 7(7) (2013) 1049-1057.

14. B. Yao, C. X. Hu, L. Lu, Adaptive Robust Precision Motion Control of a High-Speed Industrial Gantry With Cogging Force Compensations, IEEE T. Control Syst. Technol. 19(5) (2011) 1149115.

15. S. Y. Xu, Further results on adaptive robust control of uncertain time-delay systems, IET Control Theor. Appl. 2(5) (2008) 402-408. 